\title{
Is Ankylosing Spondylitis a Risk Factor of Recurrent Cerebrovascular Disease?
}

\begin{abstract}
Zeynep Özözen Ayas*
Sakarya University Training and Research Hospital, Department of Neurology, Sakarya, Turkey

*Corresponding author: Zeynep Özözen Ayas, Sakarya University Training and Research Hospital, Department of Neurology, Adnan Menderes Road, Saglık St. No: 195, 54100, Adapazarı, Sakarya, Turkey
\end{abstract}

Received: April 29, 2017; Accepted: May 25, 2017; Published: J une 01, 2017

\begin{abstract}
Rheumatological diseases causing systemic vasculitis are one the causes of stroke in young patients.AS is a systemic inflammatory rheumatological disorder that primarily involves axial skeleton. AS may cause premature atherosclerosis. In addition, small vessel inflammation may play a role in the pathogenesis of vascular disease in patients with AS. Association of AS and cerebrovascular disease is rare. Herein, we discussed the role of AS as an etiological factor for recurrent stroke in young patient.
\end{abstract}

Keywords: Rheumatological diseases; Atherosclerosis; Cerebrovascular disease; Ankylosing spondylitis

\section{Introduction}

The etiology of ischemic stroke varies in the young. Rarely, some rheumatological diseases associated with some cerebrovascular events are implicated in the etiology. It is possible to reduce the risk of recurrent stroke episodes by performing a systematic evaluation and selecting appropriate treatments tailored to risk factors and stroke etiology in young patients. Concurrence of ankylosing spondylitis (AS) and stroke is rare. Herein, we discussed the role of AS as an etiological factor for recurrent stroke in young patient.

\section{Case Presentation}

A 36-year-old woman presented to hospital with numbness in her right arm and leg. Her medical history was remarkable for ankylosing spondylitis for 8 years, for which she was taking etanercept. She also had had ischemic stroke (right-sided pontine infarction) 1 year ago and was using acetyl salicylic acid 100mg since then. Her neurological examination was notable for left hemi hypoesthesia, left hemiparesis (5-/5, sequela), and left flexor plantar response. Her vital signs were stable and her routine hemogram and biochemical tests were within normal limits. Her electrocardiogram showed normal sinus rhythm. A cranial computerized tomography (CT) did not show any acute pathology. A brain diffusion and apparent diffusion co-efficient magnetic resonance imaging (MRI) examination revealed an area of acute infarction in left corona radiata (Figure 1). Additionally, there were ischemic gliotic changes consistent with small vessel disease in subcortical white matter on both sides on T2 and FLAIR sequences of brain MRI (Figure 2). The patient was admitted to our department. An electrocardiography and a carotid vertebral Doppler angiography taken for stroke etiology were both normal. Detailed vasculitis and genetic workup were done, and the ANA titer was found 1/1000 positive in a granular cytoplasmic pattern. A carotid MR angiogram, holter, and transthoracic echocardiogram taken to identify the etiology of recurrent stroke episodes at young age were all normal. The acetyl salicylic acid dose was increased to $300 \mathrm{mg}$ and the patient was discharged to return for follow-up visits.

\section{Discussion}

Stroke in the young population constitutes $4-10 \%$ of all stroke episodes, and its incidence ranges between 9-11/100.000 [1]. Its etiology is markedly different than that in the aged population. Rheumatological conditions causing systemic vasculitis are one the causes of stroke in young patients. Polymyalgia rheumatica, takayasu, Behçet disease, and systemic lupus erythematosus are the most widely known examples. It has also been reported that, compared to the general population, the risk for stroke is higher particularly among those younger than 50 years of age [2].

AS is a systemic inflammatory rheumatological disorder that primarily involves axial skeleton but also affects peripheral joints and non-articular structures. Although literature data has shown that it is more common in men than women, our patient was a woman [3]. It mostly affects young patients, with the disease onset being most common between the ages of 20 and 30 years [3]. Consistently, our patient was diagnosed at the age of 27 years. As the condition affects young population, it must be considered in the differential diagnosis of stroke in the young. In a study where Zhang et al compared stroke patients with and without AS, patients with AS were younger than those without [4].

Inflammation is a risk factor for stroke [5]. It is pathologically seen in perforated arteriolar walls and perivascular tissue [6]. It is thought that chronic inflammation may accelerate atherosclerotic
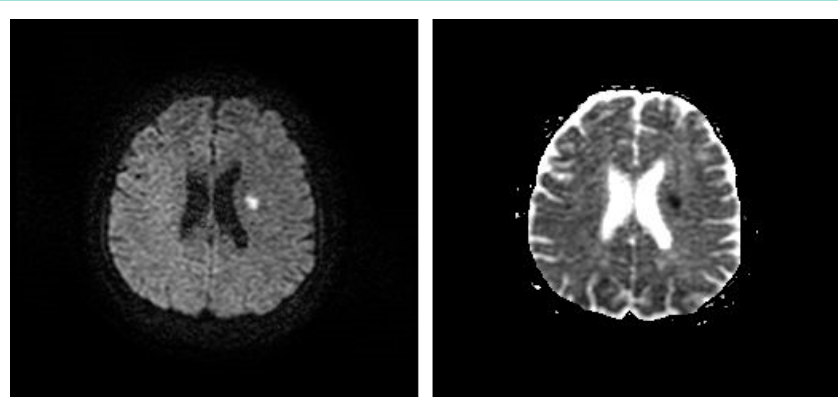

Figure 1: Diffusion and ADC MRI showed acute infarct area.
Phys Med Rehabil Int - Volume 4 Issue 2 - 2017

ISSN : 2471-0377 | www.austinpublishing group.com

Ayas. () All rights are reserved
Citation: Ayas ZÖ. Is Ankylosing Spondylitis a Risk Factor of Recurrent Cerebrovascular Disease?. Phys Med Rehabil Int. 2017; 4(2): 1115 


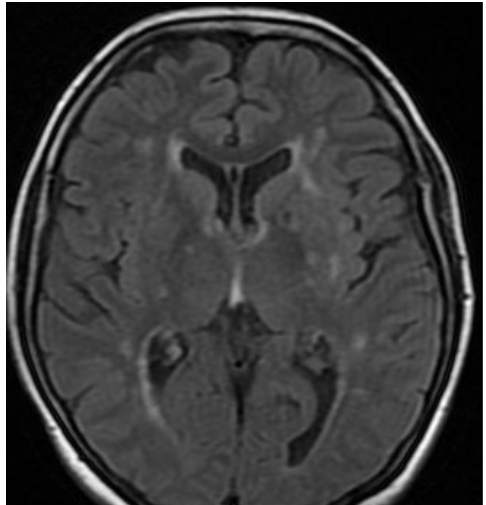

Figure 2: Ischemic gliotic changes consistent with small vessel disease in subcortical white matter on FLAIR MRI.

process in AS [7]. A study showed an association between premature atherosclerosis and chronic inflammatory condition [8]. Patients with AS are at increased risk for developing coronary artery disease [9]. One study showed that the risk of cardiovascular events was increased by $30-50 \%$ in patients with AS [10]. Endothelial injury is the initial step of atherosclerosis that underlies cerebrovascular disorders. Factors contributing to endothelial injury, such as immune complex formation and complement activation, are also seen in rheumatological conditions. In a study of patients with AS it was shown that they were at increased risk for a premature onset of cerebrovascular disorders. It has been suggested that premature atherosclerosis may be associated with AS. In addition, an increased incidence of small-vessel type of stroke in patients with AS indicates that small vessel inflammation may play a role in the pathogenesis of vascular disease [4]. Our patient similarly had ischemic gliotic areas in subcortical white matter bilaterally on MRI, suggesting that she may also have had AS-associated small vessel disease.

In all types of cerebrovascular disorders the risk of recurrence is highest in the first year and decreases gradually thereafter [11]. In line with the literature, stroke recurred one year later in our patient. One study linked stroke recurrences to transient ischemic attack, atrial fibrillation, male sex, and hypertension [12]. Detailed evaluations were done in our patient to identify the etiology of stroke. However, no abnormality was found in her electrocardiogram, carotid vertebral doppler, carotid MR angiography, holter, and transthoracic echocardiogram.

In this paper, it was discussed that AS may be assumed to play a role in the etiology of recurrent stroke in the young when no other factor can be identified; and the potential mechanisms for this association were also mentioned.

\section{References}

1. Bijen Nazlıel. Cerebrovascular Disease. In: Sevin Balkan, editor. Stroke in Young Patient. $3^{\text {rd }}$ ed. Ankara: Güneş; 2009; 375.

2. Wiseman SJ, Ralston SH, Wardlaw JM. Cerebrovascular Disease in Rheumatic Diseases: A Systematic Review and Meta-Analysis. Stroke. 2016; 47: 943-950.

3. Braun J, Sieper J. Ankylosing spondylitis. Lancet. 2007; 369: 1379-1390.

4. Zhang X, Liu R, Wang J, Zhang Y, Liu Y, Yu Z, et al. Risk of premature cerebrovascular disease in patients with ankylosing spondylitis. Acta Reumatol Port. 2016; 41: 322-327

5. Lindsberg PJ, Grau AJ. Inflammation and infections as risk factors for ischemic stroke. Stroke. 2003; 34: 2518-2532.

6. Bailey EL, Smith C, Sudlow CL, Wardlaw JM. Pathology of lacunar ischemic stroke in humans a systematic review. Brain Pathol. 2012; 22: 583-591.

7. Hamdi W, Bouaziz MC, Zouch I, Ghannouchi MM, Haouel M, Ladeb MF, et al. Assessment of Preclinical Atherosclerosis in Patients with Ankylosing Spondylitis. J Rheumatol. 2012; 39: 322-326.

8. Hahn BH, Grossman J, Chen W, McMahon M. The pathogenesis of atherosclerosis in autoimmune rheumatic diseases: Roles of inflammation and dyslipidemia. Journal of autoimmunity. 2007; 28: 69-75.

9. Peters MJL, Visman I, Nielen MMJ, Van Dillen N, Verheij RA, van der Horst-Bruinsma IE, et al. Ankylosing spondylitis: a risk factor for myocardial infarction. Ann Rheum Dis. 2010; 69: 579-581.

10. Eriksson JK, Jacobsson L, Bengtsson K, Askling J. Is ankylosing spondylitis a risk factor for cardiovascular disease, and how do these risks compare with those in rheumatoid arthritis? Ann Rheum Dis. 2017; 76: 364-370.

11. Leoo T, Lindgren A, Petersson J, Von Arbin M. Risk factors and treatment at recurrent stroke onset. Results from the recurrent stroke quality and epidemiology (RESQUE) study. Cerebrovasc Dis. 2008; 25: 254-260.

12. Jørgensen HS, Nakayama H, Reith J, Raaschou HO, Olsen TS. Neurology. Stroke recurrence: predictors, severity, and prognosis. The Copenhagen Stroke Study.1997; 48: 891-895.
Phys Med Rehabil Int - Volume 4 Issue 2 - 2017 ISSN : 2471-0377 | www.austinpublishing group.com Ayas. () All rights are reserved Rehabil Int. 2017; 4(2): 1115 . 